\title{
Vaginal cuff dehiscence after intracavitary brachytherapy for endometrial cancer
}

\author{
Kevin Albuquerque, MD, MSc, FRCS', Karan Shah, MD', Prof. Ronald Potkul, MD² \\ 'Department of Radiation Oncology, UT Southwestern Medical Center, 2Department of Obstetrics and Gynecology, Loyola University \\ Medical Center, Maywood, Illinois, USA
}

\begin{abstract} plications should be discussed with the patients.

\section{Purpose}

Vaginal cuff dehiscence and bowel evisceration is an unusual complication following standard trans-abdominal hysterectomy. However, this has been described after laparoscopic hysterectomy, including robotic laparoscopic hysterectomy [1,2]. We describe 2 cases of vaginal cuff dehiscence after intracavitary vaginal cuff high-dose rate brachytherapy.
\end{abstract}

We describe 2 unusual cases of vaginal dehiscence after intracavitary brachytherapy performed following robotic laparoscopic hysterectomy (RLH) along with their management. This unusual complication, which may be more common because of the robotic procedures, can be prevented by careful examination and possible delay of the onset of brachytherapy, if indicated. Our report reviews techniques of avoiding this complication and suggests that these com-

J Contemp Brachyther 2012; 4, 2: III-112 DOI: $10.5114 / j c b .2012 .29368$

\section{Cases}

A 59-year-old Asian and 69-year-old Caucasian females having undergone uncomplicated RLH with node dissection, presented to us separately for vaginal brachytherapy six weeks after surgery as part of their adjuvant therapy. There were no risk factors for impaired healing. A standard surgical procedure was used for the hysterectomy and colpotomy was performed with monopolar scissors, then approximated by running vicryl sutures. Both were FIGO Stage $1 \mathrm{~B}$ grade 3 . The vaginal cuff was assessed prior to the procedure and found to be intact. However, on placing the vaginal cylinder and securing it in place, it slid in more than expected with a gush of serosanguineous fluid. Planning CT scan images (Fig. 1) for patient 1 show the cylinder displaced abutting the sacrum through the vaginal dehiscence. Speculum examination revealed a complete disruption of the vaginal cuff with some bowel protruding through the disrupted cuff. This was confirmed to be a sigmoid loop at the time of repair (transvaginal approximation of the vaginal cuff with absorbable suture) performed under anesthesia. Both patients reported again for attempted brachytherapy 6 weeks after vaginal closure surgery. This was possible in one but the other had strictures with deformation of the vaginal cuff making delivery of brachytherapy impossible.

\section{Discussion}

Vaginal dehiscence following total hysterectomy has been reported rarely, with an increasing trend recently as the number of laparoscopic robotic hysterectomies increases [1,2]. At our institution we have performed a large number of such procedures since 2007 and these 2 cases were not part of our early series and in fact did not occur at the same time. This has become the preferred procedure because of the patient ease, reduced blood loss, length of hospital stay, and reduced pain.

Our report is the first one (to our knowledge) describing this complication following vaginal brachytherapy. Based on our experience it is difficult to predict which patient is prone to develop this complication. With an increased role of vaginal brachytherapy in low to intermediate risk patients with endometrial cancer [3]; this complication should be carefully anticipated. During the procedure a significant pressure results and if the cuff is not healed, this can trigger dehiscence.

Our current approach includes careful examination of the vaginal cuff with inspection and firm but gentle digital palpation to rule out small dehiscence, which could progress with pressure from cylinder insertion. We also recommend reproducible interfraction positioning of cylinder by confirmatory fluoroscopy to verify placement and proper inspection of the end of the cuff therapy for bleeding or serosanguineous discharge to rule out possible dehiscence. Using these techniques, we have not had any further 


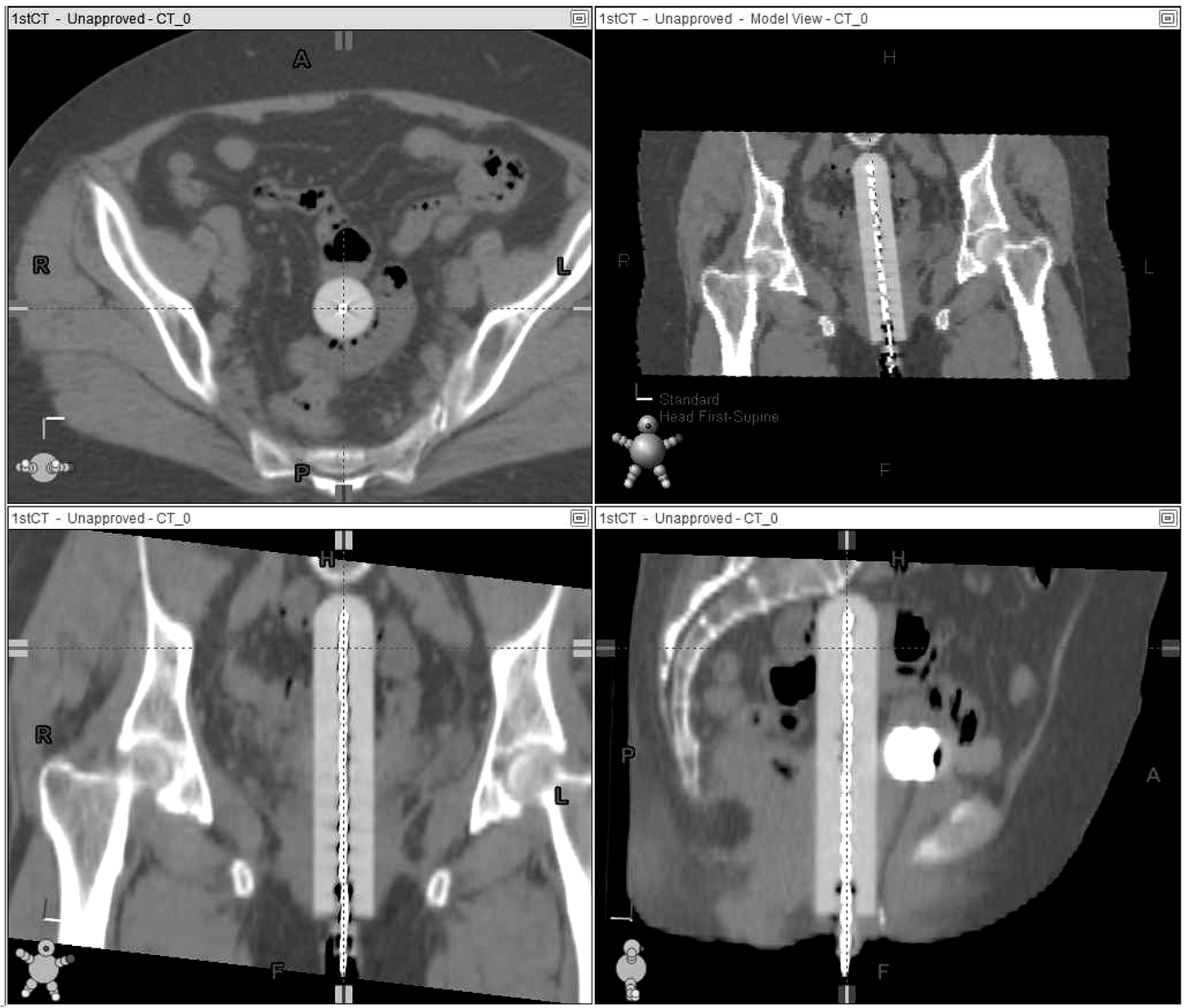

Fig. 1. Brachytherapy planning CT scan images in 3 planes show the cylinder which is abutting the sacrum having passed through the vaginal dehiscence

dehiscence. Delaying the onset of vaginal brachytherapy may be indicated if healing is suspected. Attention to meticulous suturing techniques and delayed absorbable sutures like PDS by the surgeon will also reduce the risk [4]. Patients should be informed about this possibility by the surgeon as well as the radiation oncologist and we have included this routinely in our discussion of complications for the patient.

\section{Conclusions}

Vaginal dehiscence following total hysterectomy is a rare surgical complication which may be triggered by vaginal brachytherapy because of pressure required at vaginal cuff for proper delivery of radiation. This can be successfully anticipated and avoided if the brachytherapist is aware of this happening after robotic laparoscopic surgery.

\section{References}

1. Hur HC, Donnellan N, Mansuria S et al. Vaginal cuff dehiscence after different modes of hysterectomy. Obstet Gynecol 2011; 118: 794-801.

2. Kho RM, Akl MN, Cornella JL et al. Incidence and characteristics of patients with vaginal cuff dehiscence after robotic procedures. Obstet Gynecol 2009; 114: 231-235.

3. Nout RA, Smit VT, Putter H et al. Vaginal brachytherapy versus pelvic external beam radiotherapy for patients with endometrial cancer of high-intermediate risk (PORTEC-2): an open-label, non-inferiority, randomised trial. Lancet 2010; 375: 816-823.

4. Zapardiel I, Zanagnolo V, Peiretti M et al. Avoiding vaginal cuff dehiscence after robotic oncological surgery: reliable suturing technique. Int J Gynecol Cancer 2010; 20: 1264-1267. 